\title{
Raising consciousness
}

\begin{abstract}
The national debate over Terri Schiavo exposed a critical gap between emotional fervor about brain-injured patients and the medical science that informs standards of care for them. Some of the questions raised in the public and legal forums point to a need for research and enhanced understanding of the mechanisms of recovery from disorders of consciousness.
\end{abstract}

In the aftermath of the stormy political, ethical, legal, familial, and medical controversies surrounding the sensationalized right-to-live versus right-to-die case of Terri Schiavo, we are left with heightened sensitivities to the plight of families and patients with disorders of consciousness. It is estimated that there may be as many as 15,000 patients in the United States who are in a persistent vegetative state (PVS) and more than 100,000 others who are in a minimally conscious state (MCS). Even though these cases present some of the most complicated medical and ethical issues of our time (1), families and physicians together often quietly weigh the options that surround these tragic circumstances and take appropriate actions without engagement of the entire United States government, politicians, the Supreme Court, state and local jurisdictions, clergy, protestors, special interest groups, and national and international media that emerged during the Schiavo case. Nonetheless, the frenzy that surrounded the case leaves some useful insights.

First, the essential elements of the generic landscapes that accompany severe brain injury were exposed, providing a platform on which to open a multifaceted dialogue of diverging views. Second, the current paucity of health care options and supporting research aimed toward understanding the underlying mechanisms of disorders of consciousness for this vulnerable population of patients was also exposed.

The differential diagnosis of PVS and MCS is based on the neurological examination. Patients in a PVS, like Terri Schiavo, demonstrate no signs of conscious behavior. They do, however, show spontaneous eye opening along with EEG evidence of sleep-wake cycles. These patients often do not require mechanical respiration or other life-support measures because dedicated brain stem circuits are able to sustain these functions. Further, the diagnostic criteria for PVS relies on the absence of behaviors that typically accompany conscious awareness such as sustained and reproducible, purposeful, or voluntary responses to sensory stimulation, language comprehension, or expression (2).

Patients in an MCS, on the other hand, retain limited and intermittent capacity for conscious behavior. These patients occasionally demonstrate clear-cut signs of self- or environmental awareness (3). In distinction to the diagnosis of PVS, the diagnosis of MCS is based on the presence of specific behavioral manifestations of conscious awareness. These behaviors occur inconsistently, must be differentiated reliably from reflexive, random, and spontaneous behavior, and include functions such as simple command following, production of yes/no responses, intelligible verbalization, and contingent behavioral responses such as appropriate affect, purposeful reaching, and pursuit eye movements $(2,3)$. At present, there are guishing PVS and MCS conditions.

In the specific instance of Terri Schiavo, the medical situation was unambiguous. She suffered a sustained period of anoxia following a cardiac arrest in 1990, which left her with a flat EEG and remarkable CT scans consistent with global brain damage secondary to severe anoxic injury excluding the possibility for recovery. Following extensive neurological and behavioral evaluations, the Florida Supreme Court ruled based on the medical evidence that she was in a PVS. Nonetheless, public exposure of this case raised substantial doubts about her diagnosis, cognitive status, and prognosis that eroded public confidence in the medical assessment and complicated the ethical, legal, and medical considerations of the case.

We might be tempted to attribute this controversy between medical and nonmedical opinions to layperson inexperience. However, a more self-critical view is that the dissonance between the two was due in part to the fact that medical science falls short of a comprehensive and convincing understanding of disorders of consciousness. It follows that perhaps we as physicians and no imaging procedures capable of distin- scientists have not produced a sufficient body of evidence or models to offer explanations based on an understanding of the basic mechanisms of loss of consciousness. Such a body of evidence and understanding would not have changed the situation for Terri Schiavo. However, in the case of MCS patients, we do not have answers to basic questions such as why do some patients emerge and others do not? what are the factors that predict emergence from MCS? and how can therapeutics be optimized for the individual patient?

Understanding of the mechanisms of cognitive disabilities has traditionally relied on lesion studies, where a loss of function was associated with a specific neural substrate. For example, in the late 1800s, Broca and Wernicke associated aphasias with injury to left hemisphere inferior frontal and superior temporal gyri, respectively. Currently investigations of cognitive processes frequently rely on neuroimaging studies of both nonimpaired and impaired populations of subjects. Fortunately, investigations of normal consciousness and "lesions of consciousness" are emerging as a legitimate and productive area of investigation. Recently, our research team applied functional MRI to assess the neurocircuitry that underlies receptive language functions in MCS patients and in healthy volunteers, discovering that intact neural networks capable of sustaining language functions were active in the MCS patients. However, the threshold for eliciting a response from those networks required a highly salient stimulus as opposed to lower-activation thresholds capable of driving the same networks in nonimpaired subjects (4). These findings suggest that functional neuroimaging may offer a new source of indicators to assess status and outcome predictions as well as lead to a modelbased approach to guide new therapeutics aimed toward recovery of consciousness. Yet they also raise new questions about the quality of cognition and perception experienced by MCS patients at various stages of recovery. Other technologies and investigational techniques such as PET, SPECT, and electrophysiology are also on the horizon (5), although the potential benefits of many of these efforts are also not yet sufficiently developed to apply to PVS or MCS patients. 
Research on disorders of consciousness is currently challenged by an extraordinary number of obstacles, including lack of funding initiatives; lack of provisions to allow legally authorized representatives to provide consent; inconsistent regulatory guidelines across states and institutions that complicate necessary collaborative efforts; conventional biases that categorize this population of patients as beyond help; in the case of neuroimaging studies, lack of billing codes for imaging procedures; and procedural complexities that require coordinated efforts from large numbers of collaborating specialists. These obstacles (although imposing) seem relatively minor when weighed against the potential benefits of a better understanding of brain injury and mechanisms of recovery, as well as the possible advent of informative neuroimaging, electrophysiological, and behavioral assessment techniques (6). All in all, accelerated research efforts focused on both investigations of consciousness and disorders of consciousness, as well as resolution of the many obstacles to performing the research, could bring about a "quantum leap" in advantages for informed clinical practice serving severely brain-injured patients.

One of the "take-home" bullet points from the exposure of the Schiavo case is that, in general, our understanding of disorders of consciousness falls short of our imperative to care for a large population of patients with severe brain injury. Although Terri Schiavo was, unfortunately, not a patient who would have benefited from improved assessment and therapeutic options, it can be assumed that there exists a large population of MCS patients who would directly benefit from accelerated research, improved treatment options, and dialogue regarding the medical, therapeutic, ethical, and legal issues that surround care and treatment objectives for patients with disorders of consciousness. Thus, future advances in the scientific investigation of consciousness and in the treatment of the disorders of consciousness are part of the necessary condition to assure dignified and personalized treatment of brain-injured patients absent a repeat performance of the sensationalized tragedy turned to drama as in the case of Terri Schiavo.

\section{Joy Hirsch}

fMRI Research Center, Columbia University Medical Center, New York, New York, USA. jh2155@columbia.edu

1. Fins, J.J. 2003. Constructing an ethical stereotaxy for severe brain injury: balancing risks, benefits and access [review]. Nat. Rev. Neurosci. 4:323-327.

2. Giacino, J., and Whyte, J. 2005. The vegetative and minimally conscious states: current knowledge and remaining questions. J. Head Trauma Rehabil. 20:30-50.

3. Giacino, J.T., et al. 2002. The minimally conscious state: definition and diagnostic criteria [review]. Neurology. 58:349-353.

4. Schiff, N.D., et al. 2005. fMRI reveals large-scale network activation in minimally conscious patients. Neurology. 64:514-523.

5. Laureys, S., Owen, A.M., and Schiff, N.D. 2004. Brain function in coma, vegetative state, and related disorders [review]. Lancet Neurol. 3:537-546.

6. Fins, J.J. 2005. Rethinking disorders of consciousness: new research and its implications. Hastings Cent. Rep. 35:22-24. 